See discussions, stats, and author profiles for this publication at: https://www.researchgate.net/publication/334262870

\title{
Position and Stiffness Control of One DoF Revolute Joint Using a Biphasic Media Variable Stiffness Actuator
}

Conference Paper · February 2019

DOI: $10.1109 /$ RC. 2019.00057

CITATIONS

0

5 authors, including:

Jesus Hiram Lugo Calles

Università degli Studi di Genova

8 PUBLICATIONS 7 CITATIONS

SEE PROFILE

Rezia M. Molfino

Università degli Studi di Genova

285 PUBLICATIONS 1,206 CITATIONS

SEE PROFILE

Some of the authors of this publication are also working on these related projects:

RadioRoSo View project

Project Screw-propelled locomotion View project
READS

46

Matteo Zoppi

Università degli Studi di Genova

169 PUBLICATIONS 949 CITATIONS

SEE PROFILE 


\title{
Position and stiffness control of one DoF revolute joint using a biphasic media variable stiffness actuator
}

\author{
Jesus H. Lugo*, Alessio Caligiuri, Matteo Zoppi, Giorgio Cannata and Rezia Molfino \\ PMAR Robotics \\ University of Genoa \\ Genoa, Italy- 16145 \\ *E-mail: lugocalles@dimec.unige.it
}

\begin{abstract}
At this time, several industrial processes and service tasks need safe interactions between humans and robots. This safety can be achieved using compliance design and control of mechanisms. This paper presents a compliant revolute joint mechanism using a variable stiffness actuator. The method for adapting the stiffness in the actuator includes a member configured to transmit motion that is connected to a fluidic circuit, into which a biphasic control fluid circulates. Actuator's stiffness is controlled by changing pressure of control fluid into distribution lines. The used control fluid is biphasic, composed of separated gas and liquid fractions with predefined ratio. A mathematical model of the actuator is presented, a modelbased control method is implemented to track the desired position and stiffness, and equations relating to the dynamics of the mechanism are provided. Results from force loaded and unloaded simulations and experiments with a physical prototype are discussed.
\end{abstract}

\section{INTRODUCTION}

Stiffness control in actuators is needed when a robot must perform motion tasks in the presence of humans, or when collisions with the environment are possible. For these scenarios, velocity and position should be sufficiently accurate while minimizing the risk of damaging humans, environment and itself [1]. Applications that require controllable stiffness can be identified as "robot-human interaction" and "natural dynamics adjustment" [2]. The first one is focused on having a safer and more natural interaction between the human and the machine, while the second one deals with the adjustment of natural dynamics of the mechanical system in order to have a desired natural motion to reduce the energy consumption, legged robots for example [3].

Pneumatic actuators were implemented to achieve variable stiffness in robotic devices in [4]. This approach considers the dynamic characteristics of a pneumatic cylinder as a series elastic actuator, by replacing a four-way servo valve with a couple of three-way valves, which allows the user to modulate the stiffness and the output force. Model-based force and position controllers implemented in hydraulics circuits have been researched in [5]. The taken approach allowed the system to accurately propel a single DoF mechanism as an actuator of a Stewart platform.
Biphasic media variable stiffness actuators (BMVSA) have been extensively studied in [6]-[13]. These devices were designed using pneumatic and hydraulic components. A control fluid must be used in order to transmit motion into the circuit. This fluid is composed of two different non-mixable fluids, that can be liquid and/or gas. The schemes shown in Fig. 1 belong to the biphasic fluid models, having liquid and gas fractions. The gas works as nonlinear elastic element, altering the stiffness due to pressure gas variations, while the liquid one is assumed incompressible and used to provide pressure changes and motion to the output link. Fig. 1a represents the primal embodiment of this approach, where confined gas fractions are dispersed in the liquid. As mentioned in [9], it is preferable that the gas is close to surface of the output link of the actuator, since the volume of liquid involved when an external force moves the output link and changes the pressures of the gas fractions is minimum. Fig. $1 \mathrm{~b}$ shows a design that is convenient due to its modularity, where the gas fraction is contained in hydropneumatic accumulators (HPAs) that can be easily replaced. Some implementations of the system are industrial grippers for garment handling [10]-[12], surgical robotics concepts, suspension systems for mobile platforms [9] and actuation of revolute joints [8].

Based on Fig. 1b and its notations, the actuator is composed of a double acting hydraulic cylinder $(\mathrm{Cyll})$ connected to a hydraulic circuit. This circuit includes a supply and distribution system $(\mathrm{HyS})$ and two hydraulic distribution lines ( $\mathrm{HyCl}$ and $H y C 2$ ) respectively connected to the cylinder's chambers and the insulated HPAs (ACl and $A C 2)$. The control fluid encompasses two different fluids that can be both compressible and are in proportions according to a predefined ratio; in this case, mineral oil and Nitrogen, having the second one secluded inside the accumulators. O-ring type seals (ORS) provide the translation of the piston without leakage between the chambers. Pressure and flow control valves are included in the hydraulic circuit with aim of controlling stiffness of the actuator. BMVSA can be considered to have antagonistic controlled stiffness, where two actuators with non-adaptable stiffness and nonlinear force-displacement characteristics are coupled antagonistically. Finally, it is assumed that the gas is 


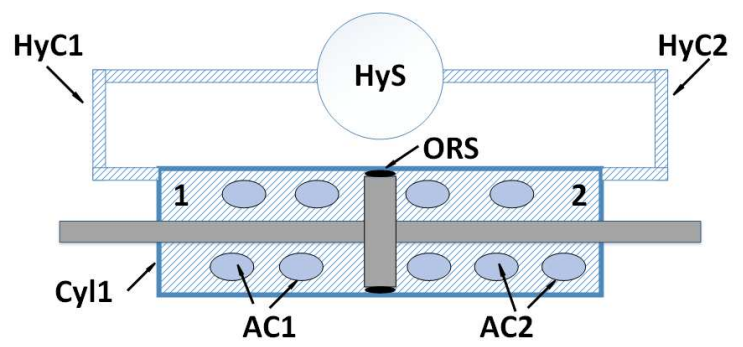

(a) Gas fraction dispersed in liquid

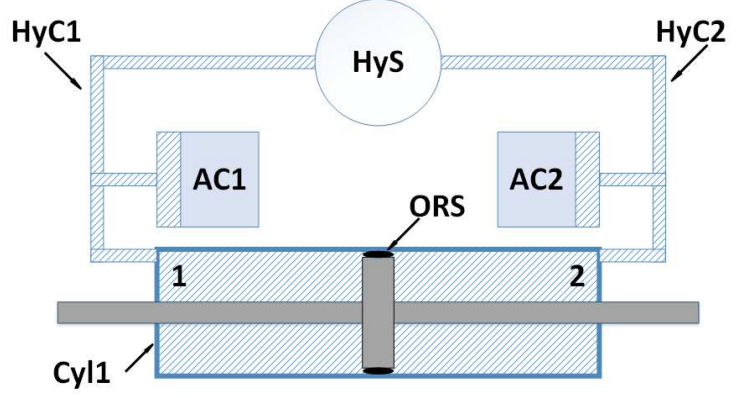

(b) Gas fraction contained in HPAs

Fig. 1: BMVSA schematics

considered ideal and the process is isothermal.

The paper is organized as follows: Section II depicts the mathematical modeling of BMVSA and proposes a modelbased controller for position and stiffness. Section III shows geometric and dynamic characteristics of the revolute joint. Section IV discusses the results from simulations of the system. Section V analyses results from experiments done with the prototype. Section VI provides conclusions and ongoing work.

\section{Modeling OF BMVSA}

The BMVSA is described as a damped harmonic oscillator in (1), where $m$ is the mass of the output link of the actuator, (piston, seals and mechanical attachments), $x_{p}$ is the displacement of the piston, $k_{v}$ is the coefficient of viscosity, $F_{p}$ is the force generated by the difference of pressures $P_{1}$ and $P_{2}$ acting on the piston's head surface $S$, as shown in (2), and $F$ is an external force. From (1), it can be stated that $F_{p}$ is taken as the restoring force of the system. The subscripts 1 and 2 denote chambers 1 and 2 and their corresponding HPAs.

$$
\begin{gathered}
m \ddot{x}_{p}+k_{v} \dot{x}_{p}-F_{p}=F \\
F_{p}=\left(P_{1}-P_{2}\right) S
\end{gathered}
$$

Considering an uniform temperature in the whole system, (3) can relate pressures and volumes.

$$
P_{i} v_{i}=C_{i}
$$

Where $v_{i}$ is the volume of gas in the $i^{t h}$ HPA and $C_{i}$ is the gas constant, which can be considered equal for both sides if the HPAs have the same characteristics $\left(C_{i}=C_{1}=C_{2}\right)$.
In (4), the volumes are expressed as the sum of added oil volumes into the hydraulic circuit $\left(v_{a i}\right)$ and the oil coming from the cylinder $\left(S x_{p}\right)$.

$$
\begin{aligned}
& v_{1}=v_{1}(0)-v_{a 1}-S x_{p} \\
& v_{2}=v_{2}(0)-v_{a 2}+S x_{p}
\end{aligned}
$$

The instantaneous stiffness $K$ is the derivative of force generated by the difference of pressures in the cylinder with respect to displacement. Equation (5) shows that stiffness is a function of $v_{a i}$. If fluid is added to both chambers, the volumes of gas reduce and the pressures increase. Similarly, if fluid is subtracted from both chambers, the volume of gas increases and the pressure in both chambers reduces.

$$
K=\frac{\partial F_{p}}{\partial x_{p}}=C S^{2}\left(\frac{1}{v_{1}^{2}}+\frac{1}{v_{2}^{2}}\right)
$$

\section{A. Model-based controller}

An encoder and a couple of pressure gauges allow to measure piston's position and pressures in chambers during the process. Equation (6) describes the volumetric flow rate, where $q_{1}$ is the moving volume in the chambers. One restriction to be considered is that subtracted/added volume cannot be larger than the total volume of gas since the liquid fraction is considered incompressible.

$$
\dot{v}_{a i}=q_{i}
$$

Equation (7) was deduced considering $u$ as spring's restoring force equal to $F_{p}$ and $x_{p}^{*}$ as the desired position, where $K_{E}$ is the characteristic gain of the spring and $u^{*}$ is the desired control signal. The error of position is stated in (8) and its derivative with respect to time in (9), where $\gamma$ is the tuning parameter to achieve minimal error.

$$
\begin{gathered}
u^{*}=-K_{E}\left(x_{p}-x_{p}^{*}\right) \\
e=u-u^{*} \\
\dot{e}=-\gamma e
\end{gathered}
$$

The error of pressure is used to control the stiffness, which is defined as the difference between the current pressures of the system and the ones related to desired stiffness $\left(P_{\text {ref }}\right)$, as shown in (10) and (11, where $\lambda$ is the tuning parameter.

$$
\begin{gathered}
e_{p}=\left(\frac{P_{1}+P_{2}}{2}-P_{\mathrm{ref}}\right) \\
\dot{e_{p}}=-\lambda e_{p}
\end{gathered}
$$

Expanding and solving (9) and (11) for $q_{i}$ allows to control the position and stiffness of the system as shown in (12), (13), (14), (15), (16) and (17).

$$
\left[\begin{array}{l}
q_{1} \\
q_{2}
\end{array}\right]=\left[\begin{array}{c}
P_{1}^{2} \\
-P_{2}^{2}
\end{array}\right] D b_{x p}(t)+\left[\begin{array}{c}
P_{1}^{2} \\
P_{2}{ }^{2}
\end{array}\right] D b_{p}(t)
$$




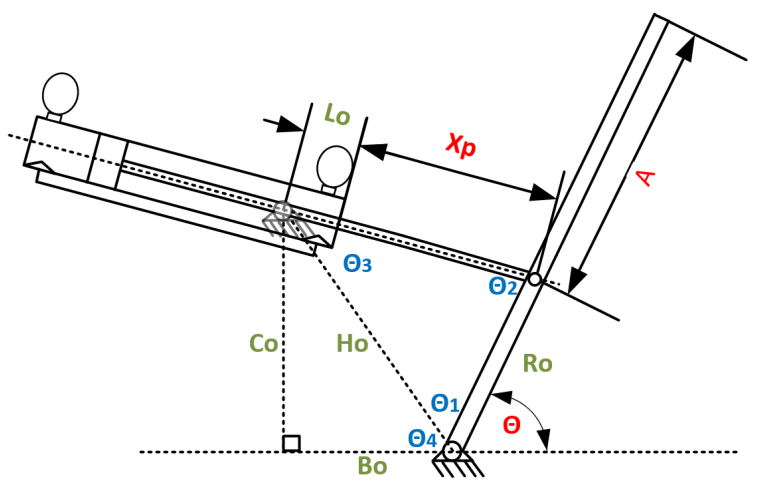

Fig. 2: Diagram of revolute joint mechanism

$$
\begin{gathered}
D=\frac{1}{P_{1}^{4}+P_{2}{ }^{4}} \\
b_{x p}(t)=P_{1}{ }^{2} q_{1}-P_{2}{ }^{2} q_{2} \\
b_{x p}(t)=\frac{C}{S}\left(-\gamma e+w_{x p}(t)\right) \\
b_{p}(t)=-2 C\left(\lambda e_{p}+w_{p}(t)\right) \\
w_{x p}(t)=-\frac{S^{2} \dot{x_{p}}}{C}\left(P_{1}{ }^{2}+P_{2}{ }^{2}\right)+\dot{u}^{*} \\
w_{p}(t)=\frac{S^{2} \dot{x_{p}}}{2 C}\left(P_{1}{ }^{2}+P_{2}{ }^{2}\right)-\dot{P}_{\text {ref }}
\end{gathered}
$$

Based on the previous equations, an array of hydraulic proportional flow valves are used to the vary the pressures in both chambers.

\section{ReVOlute JOINT}

\section{A. Geometric model of mechanism}

A revolute joint mechanism has been designed to visualize the effect of the stiffness variation of the actuator, as shown Fig. 2. This mechanism has one DoF and its geometric parameters are given in Tab. I. Equations (18) and (19) relate linear displacement $(x p)$ with angular displacement $(\theta)$.

$$
\begin{gathered}
\cos \left(\theta_{1}\right)=\frac{C_{o}}{H_{o}} \sin (\theta)+\frac{B_{o}}{H_{o}} \cos (\theta) \\
x_{p}+L_{o}=\left(R_{o}^{2}+H_{o}^{2}-2 R_{o} H_{o} \cos \left(\theta_{1}\right)\right)^{\frac{1}{2}}
\end{gathered}
$$

\section{B. Stiffness analysis}

The output link works as a rotational actuator. Its torque, called $\tau_{\text {joint }}$, is produced by $F_{p}$. Equations (20), (21), and (22) provide its definition.

$$
\tau_{\text {joint }}=\left(S C R_{o}\right)\left(\frac{1}{v_{1}}-\frac{1}{v_{2}}\right) \sin \left(\theta_{2}\right)
$$

$$
\begin{gathered}
\sin \left(\theta_{2}\right)=\frac{H_{o}}{x_{p}+L_{o}}\left(\frac{B_{o}}{H_{o}} \sin (\theta)+\frac{C_{o}}{H_{o}} \cos (\theta)\right) \\
\tau_{\text {joint }}=\tau_{w}+\tau_{I}+\tau_{F_{\text {ext }}}
\end{gathered}
$$

$\tau_{w}$ is the torque generated by the weight of the equivalent mass, given by $m_{e q}=m_{\text {Load }}+m_{\text {Link }}$, at the center of mass $\left(R_{m_{e q}}\right)$ on the output link, as shown in (23) and (24).

$$
\tau_{w}=m_{e q} g R_{m_{e q}} \sin \left(\frac{\pi}{2}-\theta\right)
$$

$$
R_{m_{e q}}=\left[m_{\text {Load }}\left(R_{o}+\frac{A_{o}}{2}\right)+m_{\text {Link }}\left(\frac{R_{o}}{2}\right)\right] \frac{1}{m_{e q}}
$$

$\tau_{I}$ is the torque caused due to the inertia of the equivalent mass, as defined in (25). An external force has been considered to be applied at the end of the output link, producing $\tau_{F_{\mathrm{ex}}}$, as shown in (26).

$$
\begin{gathered}
\tau_{I}=\frac{m_{e q} g\left(R_{m_{e q}}\right)^{2} \ddot{\theta}}{3} \\
\tau_{F_{\mathrm{ext}}}=F_{\mathrm{ext}}\left(R_{o}+A_{o}\right)
\end{gathered}
$$

The instantaneous rotational stiffness $K(\theta)$ of the mechanism is the derivative of $\tau_{\text {joint }}$ with respect to the $\theta$, as defined in (27).

$$
K(\theta)=\frac{\partial \tau_{\text {joint }}}{\partial \theta}
$$

The previous equations demonstrate that $K(\theta)$ is a function of the output force $\left(F_{p}\right)$ and the angular displacement $(\theta)$, and it is affected by the weight of the equivalent mass.

\section{Simulation}

The following simulation was performed to study the stiffness and position of the model using the parameters given in Tab. II. Two types of tests were performed: the first did not have an external force applied, while the second one had a force signal with a square wave shape with $10 \mathrm{~N}$ amplitude and baseline on $0 \mathrm{~N}$ at $0.2 \mathrm{~Hz}$. Both types of simulations were executed including the $m_{\text {Load }}$ and $m_{\text {Link }}$. Position in the actuator is measured from left end of the cylinder to its right end.

TABLE I: Geometric parameters

\begin{tabular}{ll}
\hline \hline Parameter & Length (m) \\
\hline$L_{o}$ & 0.07 \\
$C_{o}$ & 0.1 \\
$B_{o}$ & 0.105 \\
$H_{o}$ & 0.145 \\
$R_{o}$ & 0.12 \\
$A$ & 0.245 \\
$x_{p} \max$ & 0.15 \\
$x_{p} \min$ & 0 \\
\hline
\end{tabular}


TABLE II: Simulation parameters

\begin{tabular}{ll}
\hline \hline Parameter & Value \\
\hline$m$ & $1 \mathrm{Kg}$ \\
$m_{\text {Load }}$ & $3.8 \mathrm{Kg}$ \\
$m_{\text {Link }}$ & $1 \mathrm{Kg}$ \\
$K_{v}$ & $0.5 \mathrm{Ns} / \mathrm{m}$ \\
$K_{E}$ & $3 \times 10^{3} \mathrm{~N} / \mathrm{m}$ \\
$S$ & $3.776 \times 10^{-4} \mathrm{~m}^{2}$ \\
$C$ & $300 \mathrm{Pam}^{3}$ \\
$\gamma$ & $-5 \times 10^{2}$ \\
$\lambda$ & $-1.3 \times 10^{3}$ \\
$x_{p}(0)$ & $0.075 \mathrm{~m}^{-4} \mathrm{~m}^{3}$ \\
$v_{i}(0)$ & $1.5 \times 10^{-4}$ \\
\hline
\end{tabular}

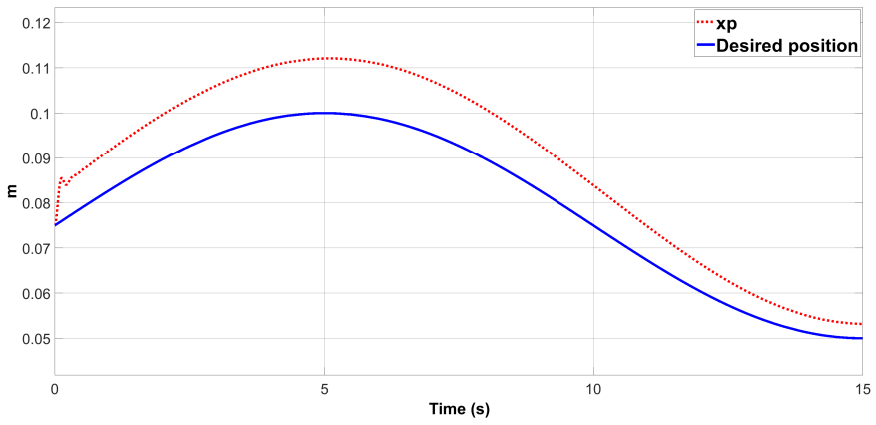

(a) Without external force

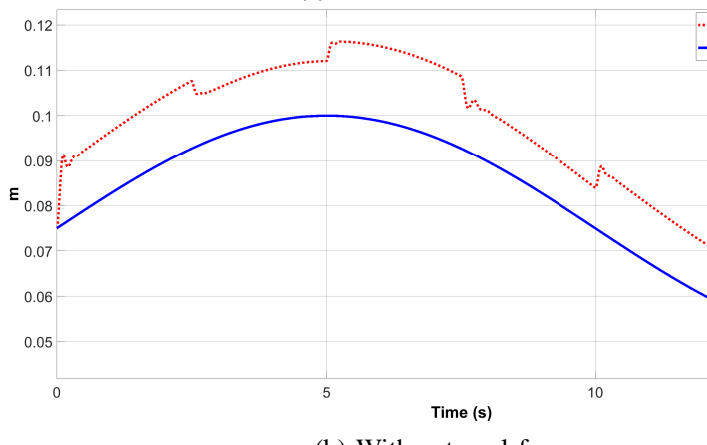

(b) With external force

Fig. 3: Position tracking in BMVSA

The desired position is given as a sinusoidal signal with 0.23 radians amplitude and baseline on 1.23 radians at 0.05 $\mathrm{Hz}$ (to approximate a linear displacement in the cylinder with amplitude of $0.025 \mathrm{~m}$ and a baseline on $0.075 \mathrm{~m}$ ), as shown in Figs. 3 and 4, while the desired stiffness is a sinusoidal signal with $20 \mathrm{Nm} /$ radians amplitude and baseline on $80 \mathrm{Nm}$ /radians at $0.1 \mathrm{~Hz}$, as depicted in Fig. 5 .

The results of the simulations prove that the model-based controller is able to properly track position and stiffness even if the desired signals to follow are nonlinear, as illustrated in Figs. 4 and 5. It is worth to note that the controller was tuned to prioritize stiffness tracking over position tracking as displayed in Fig. 3 and 4, where the desired position in never reached, while the desired mechanism stiffness is always obtained, see Fig. 5, 6 and 7. It can be stated that whenever a load force differs from zero a displacement in the actuator is generated, which is directly related to the value of $K_{E}$.

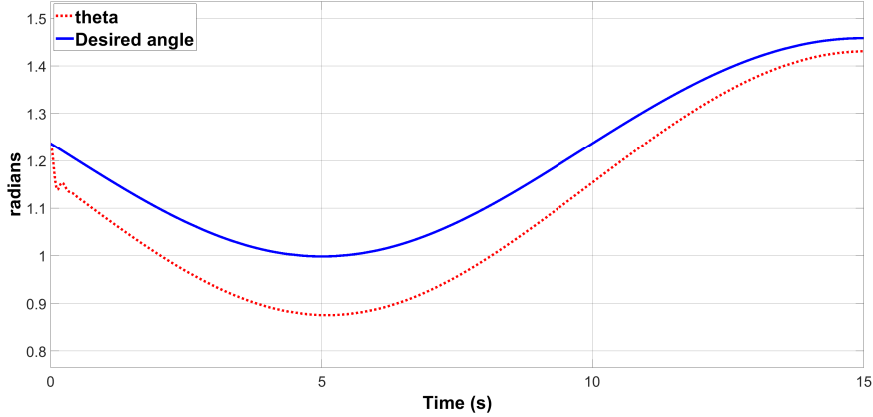

(a) Without external force

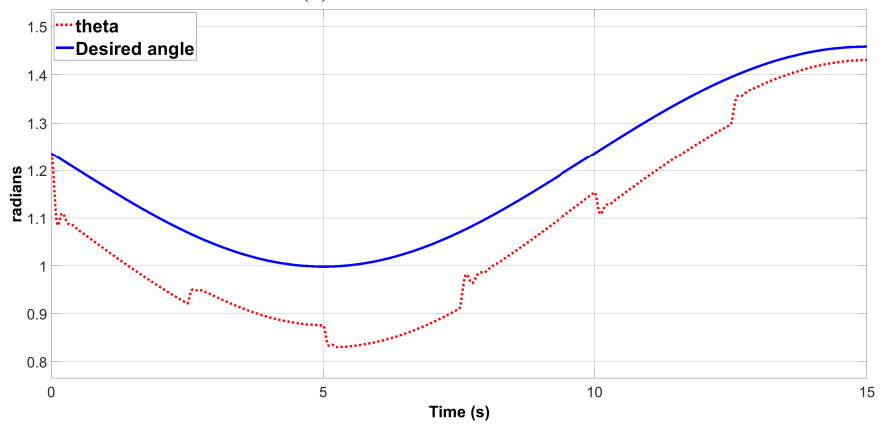

(b) With external force

Fig. 4: Angle tracking in mechanism

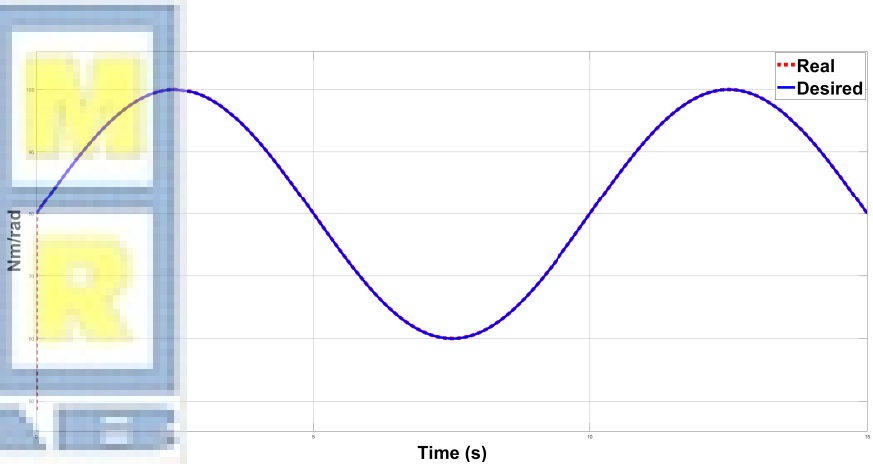

(a) Without external force

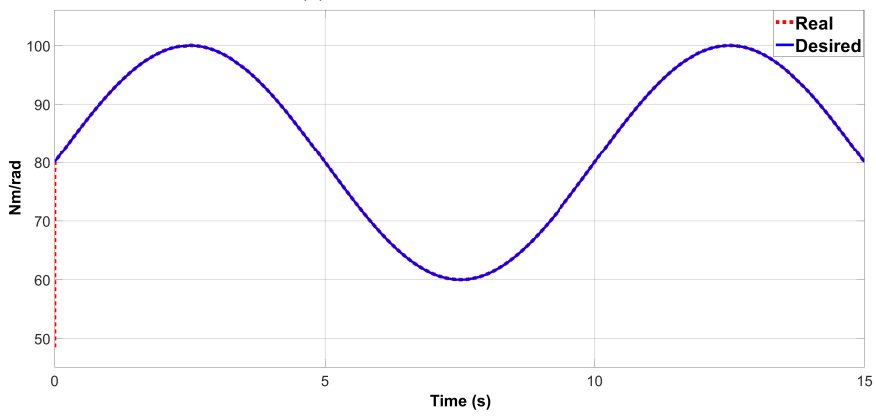

(b) With external force

Fig. 5: Stiffness tracking in mechanism

\section{EXPERIMENTS WITH THE PROTOTYPE}

The BMVSA and the mechanism have been prototyped and they are currently in testing phase. The mechanism is made of steel and it keeps geometry and sizes mentioned in Tab. I. The compression ratio $\left(\mathrm{Co}_{R}\right)$ is a physical charac- 


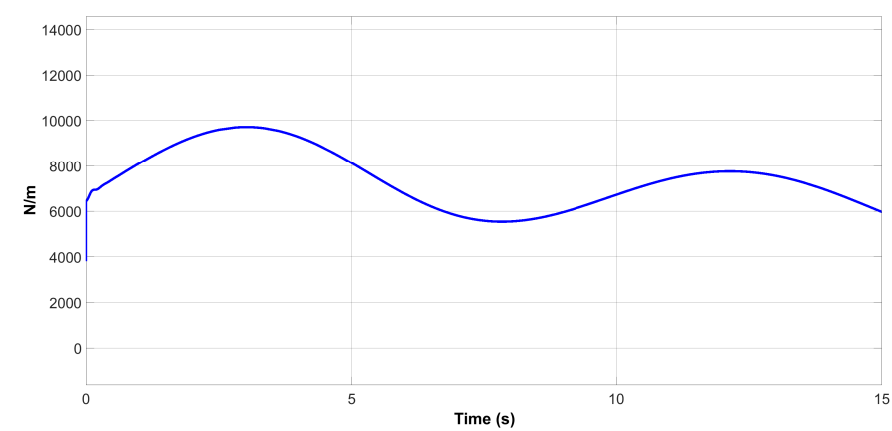

(a) Without external force

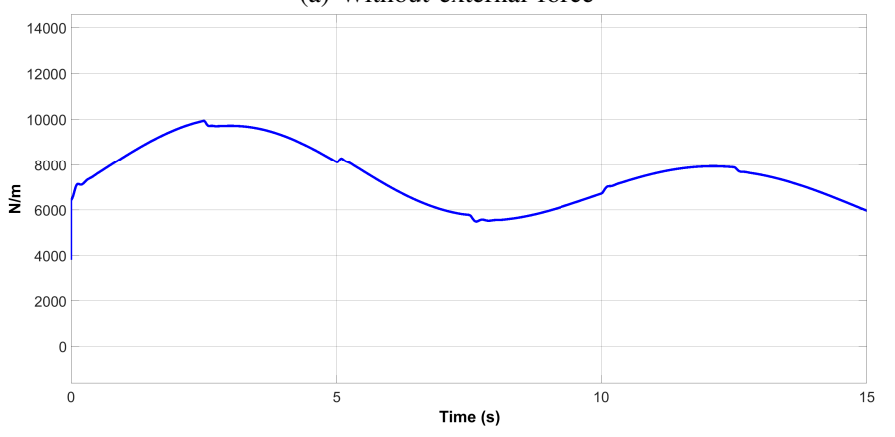

(b) With external force

Fig. 6: Stiffness tracking in BMVSA

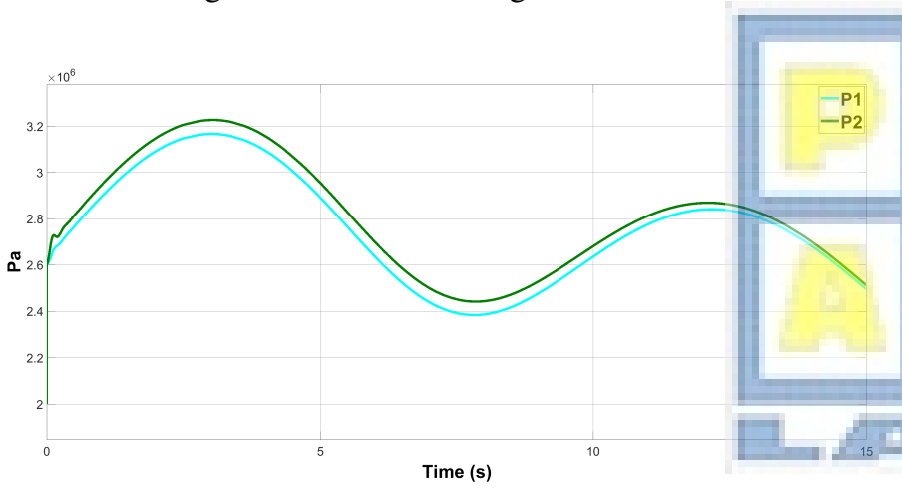

(a) Without external force

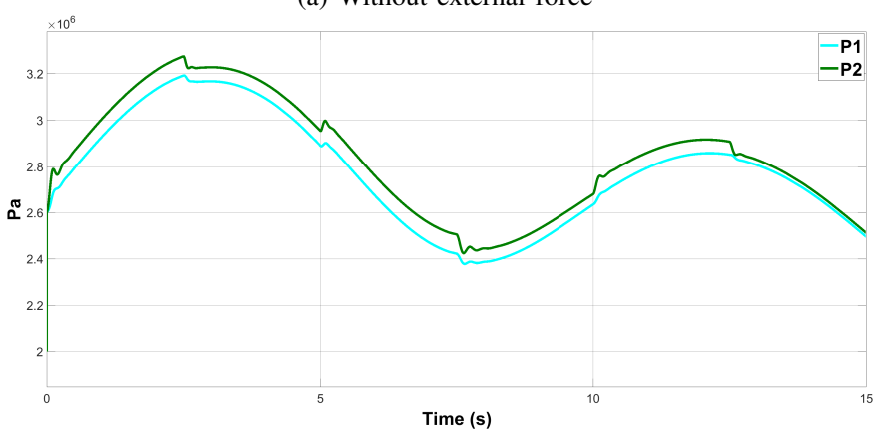

(b) With external force

Fig. 7: Pressures in BMVSA

teristic of HPA that relates the maximum pressure that the accumulator can hold to its initial pressure before its rubber diaphragm wears out considerably. Figure 8 shows a schematic of the implemented hydraulic circuit. Its main components are the hydraulic cylinder (Cyll), a pair of HPAs (ACl-2),

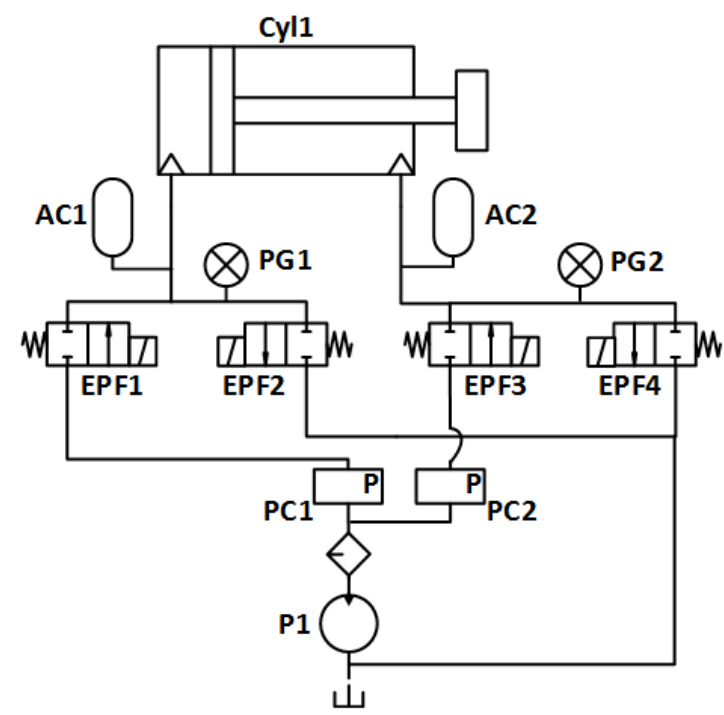

Fig. 8: Hydraulic system schematic

a hydraulic pump (HyS), two pressure gauges $(P G l-2)$, four electro-proportional flow control valves (EPF1-4) and two proportional pressure control valves (PC1-2). The purpose of having two pressure control valves is to isolate the hydraulic input for each chamber of the cylinder. The maximum flow, the maximum working pressure, the maximum difference of pressures between input and output and PWM frequency (10 liters/second, $350 \mathrm{bar}, 15 \mathrm{bar}$, and $120 \mathrm{~Hz}$, respectively for the selected valves) are $E P F$ 's performance characteristics that limit the actuator to work within their physical limits. Fig. 9 shows the prototype in unloaded state.

The previously described model-based controller provides a solution for $q_{1}$ and $q_{2}$; to obtain the desired flows, a set of four EPF is used, two per chamber. The position of the piston is measured via encoder and the stiffness is calculated in real-time using the pressure gauges. The tuning parameters and initial conditions are given in Table III. It is worth to mention that a pin shaped force gauge $(F G)$ is used as the rotation pin that connects the cylinder and the arm. $F G$ will be used for measuring the output force of the actuator in future experiments. A custom-designed control board powered by a multi-threaded ARM microprocessor is used to run the controller on real-time. This board contains drivers for valves running at $8 \mathrm{~Hz}$ for each of $E P F$.

Only loaded experiments were performed. A $3.8 \mathrm{~kg}$ metal bar is used to load the output link. Due to prototype's physical

TABLE III: Experiment parameters

\begin{tabular}{ll}
\hline \hline Parameter & Value \\
\hline$K_{v}$ & $0.5 \mathrm{Ns} / \mathrm{m}$ \\
$K_{E}$ & $2 \times 10^{4} \mathrm{~N} / \mathrm{m}$ \\
$\gamma$ & $-6.5 \times 10^{3}$ \\
$\lambda$ & $-2.5 \times 10^{3}$ \\
$x_{p}(0)$ & $0.075 \mathrm{~m}$ \\
$C o_{R}$ & 4 \\
\hline
\end{tabular}




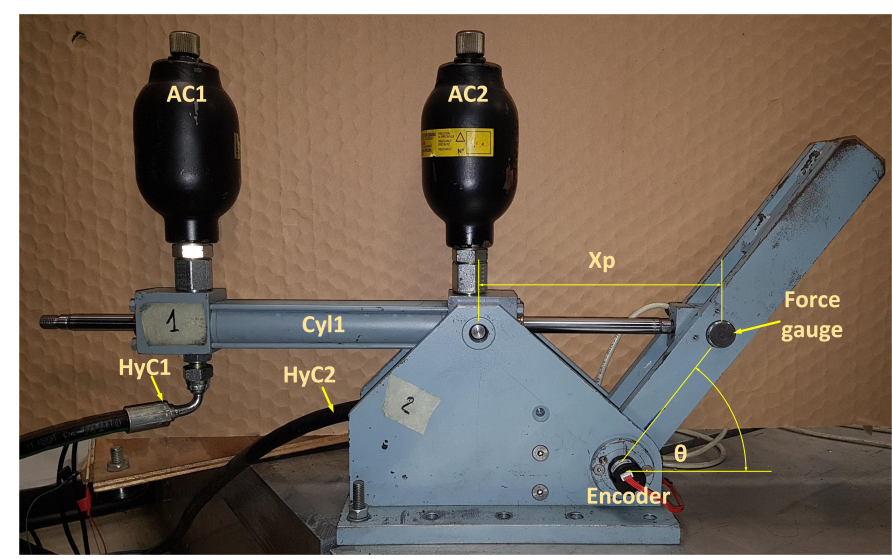

Fig. 9: Prototype of mechanism

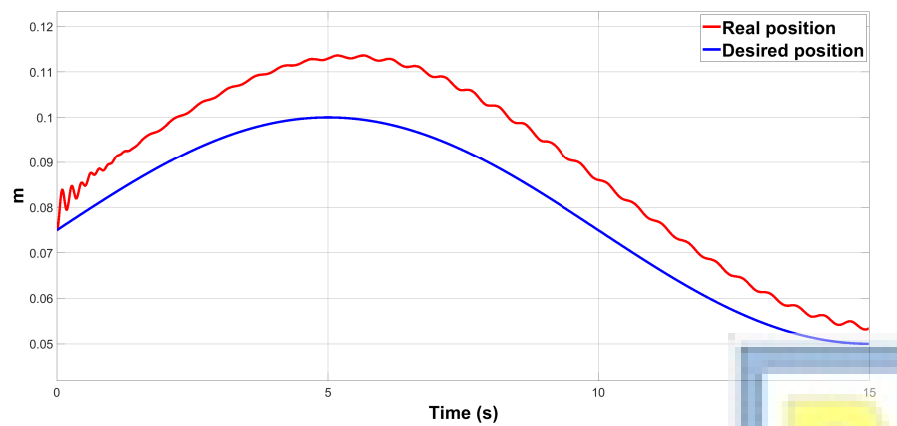

Fig. 10: Position tracking during experimen

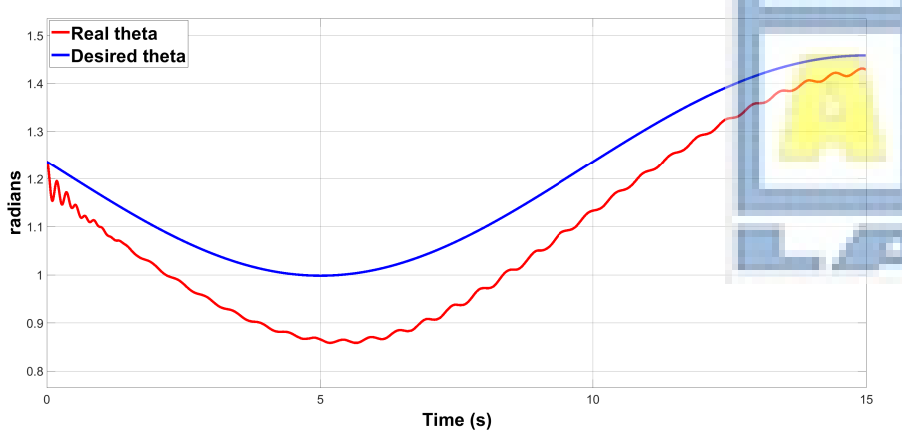

Fig. 11: Angle tracking during experiment

features, the desired position is given as a sinusoidal signal with 0.5 radians amplitude and baseline on 1.2 radians at 0.05 $\mathrm{Hz}$, while the desired stiffness is a sinusoidal signal with 20 $\mathrm{Nm} /$ radians amplitude and baseline on $80 \mathrm{Nm} /$ radians at 0.1 $\mathrm{Hz}$.

The results of the experiments confirm that the model-based controller can track position and stiffness, as depicted in Figs. $10,11,12$ and 13. Like in the simulations, the controller prioritized stiffness tracking over position tracking. Another presented phenomena that appear during the experiments are small jumps in the position, as shown 10 and 11, these are related to the static friction of the piston, the friction in mechanism joints, and the low frequency in which the EPFs work.

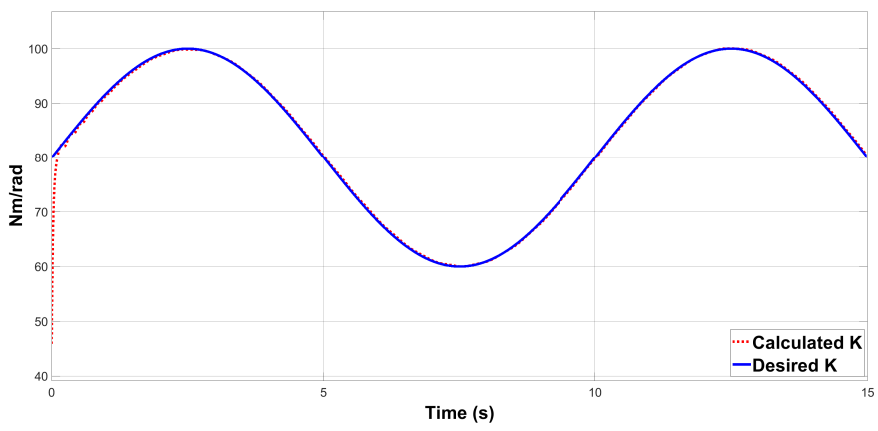

Fig. 12: Rotational stiffness tracking during experiment

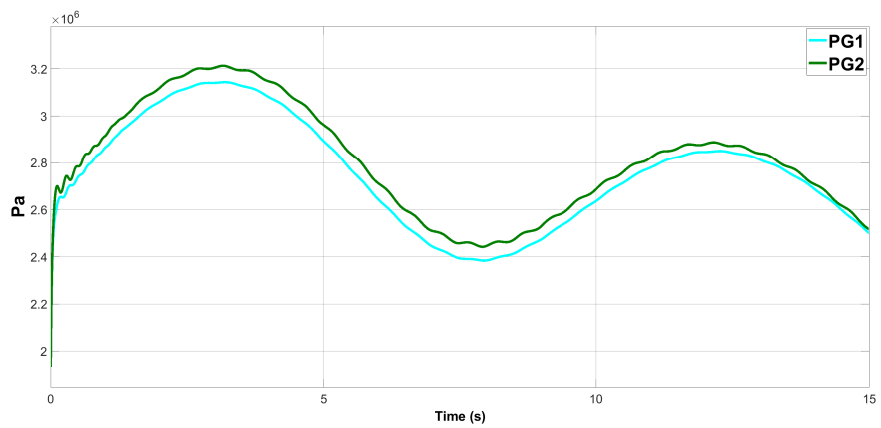

Fig. 13: Measurements of pressure gauges

\section{CONCLUSION}

The stiffness of the BMVSA is related to the pressures of the gas fractions contained in the accumulators. This device has two advantages due to its biphasic nature: the hydraulic system gives it the possibility to have a high output force, while the nonlinearity of the gas provides a wider range of stiffness than the VSAs using traditional elastic components.

The results obtained from simulations demonstrate that the model-based controller approach can accurately track desired stiffness and approximate the desired position, while an external force acts on it. The measurements taken during the experiments corroborate the previously mentioned about the simulations, pointing out the influence of the output link's mass, friction and load's inertia.

Momentum-based collision detection methods [14] and reaction algorithms are being tested in the system to increase the safety during physical interaction. The monitoring of collisions is done using residuals of the momentum of the mechanism without knowledge of the joint torque or stiffness. The reaction algorithms allow the robot to stop its motion and to reduce its stiffness for the sake of safety of its structure and the colliding object.

\section{REFERENCES}

[1] B. Vanderborght, A. Albu-Schaffer, A. Bicchi, E. Burdet, D. G. Caldwell, R. Carloni, M. Catalano, O. Eiberger,W. Friedl, G. Ganesh, et al. "Variable impedance actuators: A review," Robotics and autonomous systems, vol. 61 (12), pp. 1601-1614, 2013.

[2] R. Van Ham, T. G. Sugar, B. Vanderborght, K. W. Hollander, and D. Lefeber, "Compliant actuator designs", IEEE Robotics and Automation Magazine, vol. 16 (3), 2009. 
[3] C. Semini, N. Tsagarakis, E. Guglielmino, F. Cannella, and D.G. Caldwell, "Design of $H y Q-a$ hydraulically and electrically actuated quadruped robot," Proceedings of the Institution of Mechanical Engineers, Part I: Journal of Systems and Control Engineering, vol 225 (6), 2011.

[4] X. Shen, and M. Goldfarb, "Simultaneous Force and Stiffness Control of a Pneumatic Actuator", Journal of Dynamic Systems, Measurement, and control, vol. 129, pp. 425-434, 2007.

[5] I. Davliakos, and E. Papadopoulos, "Development of a Model-Based Nested Controller for Electrohydraulic Servos," Proceedings of the 2005 IEEE International Symposium on, Mediterrean Conference on Control and Automation Intelligent Control, pp. 107-112, 2005.

[6] M. Zoppi, "Method for adapting stiffness in a variable stiffness actuator," US Patent 8991 169, March 31, 2015.

[7] B. Han, M. Zoppi, and R. Molfino, "Variable impedance actuation using biphasic media," Mechanism and Machine Theory, vol. 62, pp. 1-12, 2013.

[8] B. Han, M. Zoppi, and R. Molfino, "Variable impedance actuation mesomicro physical models and rotation link using biphasic media," Procedia Engineering, vol. 64, pp. 1020-1029, 2013.

[9] B. Han, "Variable impedance actuation using biphasic media," Ph.D. Thesis dissertation, University of Genoa, 2013.

[10] L. Le, M. Zoppi, M. Jilich, R. Camoriano, D. Zlatanov, and R. Molfino, "Development and analysis of a new specialized gripper mechanism for garment handling," in ASME 2013 International Design Engineering Technical Conferences and Computers and Information in Engineering Conference, pp. V06BT07A013-V05BT08A023, 2013.

[11] L. Le, M. Zoppi, M. Jilich, B. Han, D. Zlatanov, and R. Molfino, "Application of a biphasic actuator in the design of a robot gripper for garment handling," in ASME 2014 International Design Engineering Technical Conferences and Computers and Information in Engineering Conference, pp. V05BT08A022-V05BT08A022, 2014.

[12] L. Le, M. Zoppi, M. Jilich, B. Han, D. Zlatanov, and R. Molfino, "Application of a biphasic actuator in the design of the clopema robot gripper," Journal of Mechanisms and Robotics, vol. 7, pp. 011011(1-8), 2015.

[13] J.H. Lugo, G. Cannata, M. Zoppi, and R. Molfino, "Modeling and Simulation of a Biphasic Media Variable Stiffness Actuator," International Journal of Mechanical Engineering and Robotics Research, 2018

[14] F. Flacco, A. De Luca, I. Sardellitti, and N. Tsagarakis, "On-line estimation of variable stiffness in flexible robot joints," The International Journal of Robotics Research, vol 31 (13), pp. 1556-1577, 2012 